\title{
FUNDAMENTALS AND APPLICATIONS OF SPECTROSCOPIC ELLIPSOMETRY
}

\author{
Débora Gonçalves* and Eugene A. Irene \\ University of North Carolina at Chapel Hill, Department of Chemistry, CB\#3290, 27599-3290 Chapel Hill - NC - USA
}

Recebido em 1/8/01; aceito em 8/11/01

\begin{abstract}
This paper describes the use of ellipsometry as a precise and accurate technique for characterizing substrates and overlayers. A brief historical development of ellipsometry and the basic principles necessary to understand how an ellipsometer works are presented. There are many examples of studies performed in addressing materials science issues, and several are presented here: measurements of thickness, optical properties, and modeling of surface roughness. These selected results obtained in our laboratory for substrates, $\mathrm{Si} / \mathrm{SiO}_{2}$ interfaces, and polymers provide evidence that ellipsometry can play a critical role in characterizing different types of materials.
\end{abstract}

Keywords: ellipsometry; polarization; films.

\section{BRIEF HISTORICAL OVERVIEW}

Ellipsometry (reflection ellipsometry) measures the changes in the state of polarization of light upon reflection from a surface. The fact that changes are measured rather than the absolute intensity of light renders ellipsometry sensitive to submonolayer surface coverage. As a non-invasive and non-destructive tool, ellipsometry requires only a low-power light source and, consequently, it does not affect most processes, which renders ellipsometry a convenient tool for in situ studies ${ }^{1,2}$. P. Drude ${ }^{3}$ (1863-1906) was the first to build an ellipsometer even before Rothen has coined the word 'ellipsometry' in $1945^{4}$. The equipment built by Drude is surprisingly very similar to many types of instruments in use today. Except for sparse papers, this technique received little attention for several decades after Drude, but in the 1970's and 1980's, ellipsometry became widely utilized, and relevant technical papers ${ }^{5-7}$ and a classical book ${ }^{8}$ were produced during this period. In 1991, Azzam compiled a considerable number of papers on ellipsometry ${ }^{9}$.

As a search of the literature indicates, the number of papers on experimental improvement of ellipsometers has been decreasing in the last years, because many of the problems that existed before the advance of computers have been solved, including measurement speeds and spectral range. Most of the equipment used today is automated with wide band light sources, scanning monochromators, optical multichannel analyzers, and interfaces with acquisition and analysis software. Early studies on ellipsometry were performed using single wavelength equipment, usually a wide band visible light source with a filter, and later lasers. However, spectroscopic ellipsometers are commonly used nowadays in different research fields.

\section{BASIC CONCEPTS}

A description of ellipsometry as an optical technique would not be complete without mentioning J.C. Maxwell (1831-1879). Maxwell's theory predicts that light is a wave represented by two mutually perpendicular vectors: $\mathbf{E}$, the amplitude of the electric field strength, and $\mathbf{B}$, the amplitude of the magnetic field strength, and

\footnotetext{
* e-mail: gdebora@if.sc.usp.br

Permanent address: Instituto de Física de São Carlos, Universidade de São

Paulo, CP 369, 13560-970 São Carlos - SP.
}

both $\mathbf{E}$ and $\mathbf{B}$ are also perpendicular to the direction of propagation, z. The electromagnetic wave is described by its amplitude and frequency in complex form:

$\mathbf{E}=\mathbf{E}_{0} \mathrm{e}^{i(\mathrm{qz}-\omega \mathrm{t})}$,

where $\mathbf{E}_{0}$ represents the maximum amplitude of $\mathbf{E}$ that propagates into the $+z$ direction and, $\omega$ is the angular frequency $(\omega=2 \pi f)$, $t$ is time, and $\mathrm{q}$ is the wave number $(\mathrm{q}=2 \pi / \lambda)$ and magnitude of the propagation vector, $\mathbf{q}(\mathbf{q}=(2 \pi / \lambda) \mathbf{z})$. After including the phase term equation (1) becomes:

$\mathbf{E}=\mathbf{E}_{0} \cos (\mathrm{qz}-\omega \mathrm{t}+\delta)$,

The vector $\mathbf{E}$ is represented by a superposition of the two components, $\mathrm{E}_{\mathrm{x}}$ and $\mathrm{E}_{\mathrm{y}}$ that are orthogonal to the direction of propagation $+\mathrm{z}\left(\mathrm{E}_{\mathrm{z}}=0\right)$ :

$\mathrm{E}_{\mathrm{x}}=\mathrm{E}_{01} \cos \left(\mathrm{qz}-\omega \mathrm{t}+\delta_{\mathrm{x}}\right) \quad \mathrm{E}_{\mathrm{y}}=\mathrm{E}_{02} \cos \left(\mathrm{qz}-\omega \mathrm{t}+\delta_{\mathrm{y}}\right)$,

where $\delta_{x}$ and $\delta_{y}$ are phase constants, and $\mathrm{E}_{01}$ and $\mathrm{E}_{02}$, amplitudes of the components $(\mathrm{x}, \mathrm{y})$ of $\mathbf{E}$.

The variation with time of the orientation of $\mathbf{E}$ along the propagation direction at a fixed location is called polarization. Any ordinary source of light is known as unpolarized because $\mathbf{E}$ oscillates randomly in the plane perpendicular to the propagation direction (note that the polarization state of light is represented only by $\mathbf{E}$ because it is a larger effect as compared with $\mathbf{B})^{10}$.

There are several ways to produce polarized light such as by dichroism (selective absorption), reflection from a surface, birefringence, and scattering. One of the most common ways of producing linearly polarized light from unpolarized light is by using a polarizer. While the polarizer transmits the $\mathbf{E}$ component oscillating in the perpendicular direction in which the film was stretched (transmission axis, TA), the light wave whose $\mathbf{E}$ is perpendicular to the TA is absorbed or attenuated ${ }^{11,12}$. To verify the state of polarization of light after passing the polarizer, a second polarizer is necessary (analyzer), and no light is transmitted through the two crossed polarizers as shown in Figure 1.

Consider in Figure 2 light with two arbitrary azimuths propagating in the $+\mathrm{z}$ direction. If $\delta\left(\delta=\delta_{\mathrm{y}}-\delta_{\mathrm{x}}\right)$ is a multiple of $\pm \pi(\delta$ 


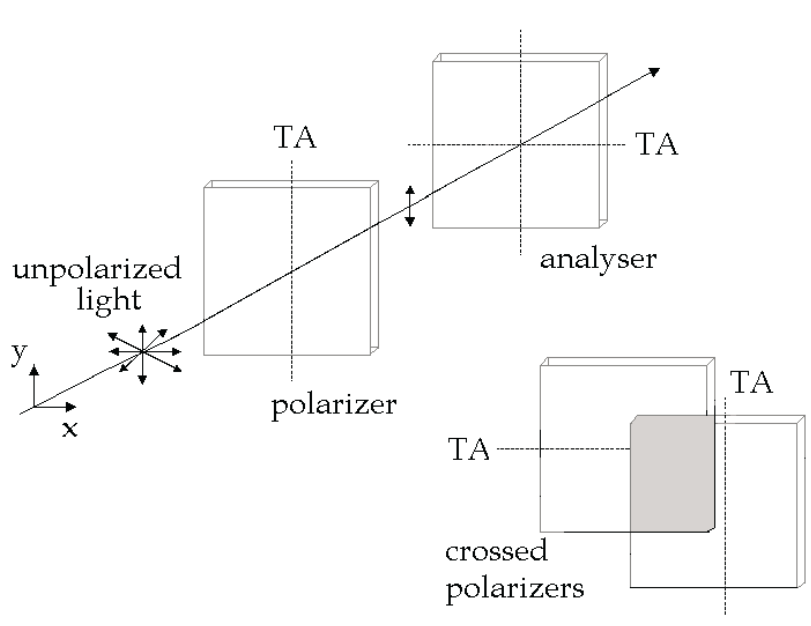

Figure 1. A linear polarizer, an analyzer, and two crossed polarizers with no light transmitted through the analyzer

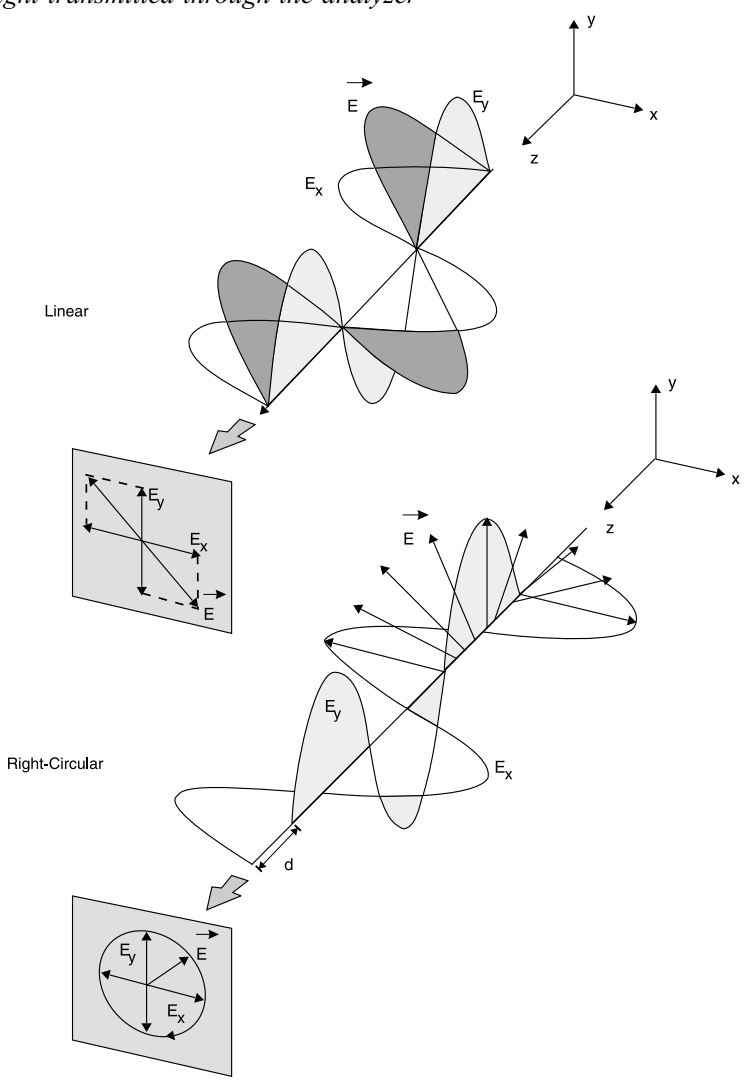

Figure 2. Linearly and right-circularly polarized lights

$=\mathrm{m} . \pi ; \mathrm{m}=0, \pm 1, \pm 2, \ldots)$, the propagating wave is linearly polarized. If the amplitudes of the $\mathrm{E}_{\mathrm{x}}$ and $\mathrm{E}_{\mathrm{y}}$ components are equal $\left(\mathrm{E}_{01}=\mathrm{E}_{02}=\right.$ $\left.\mathrm{E}_{0}\right)$, and $\delta$ is a multiple of $\pm \pi / 2(\delta=\mathrm{m} . \pi / 2 ; \mathrm{m}=0, \pm 1, \pm 2, \ldots)$, the wave is called right-circularly polarized or left-circularly polarized when $\mathbf{E}$ rotates clockwise or counterclockwise, respectively ${ }^{10-12}$. In the case of elliptically polarized light, the resultant $\mathbf{E}$ rotates and changes in magnitude in such a way that the locus of ends of the vectors traces an ellipse.

The elliptically polarized light can be produced by reflection from a flat surface or using polarizer/compensator elements. With reflection is associated a change of phase of the components of $\mathbf{E}$ parallel $\left(\mathrm{E}_{p}\right)$ and perpendicular $\left(\mathrm{E}_{s}\right)$ to the plane of incidence of light.
With the use of a compensator (also called retarder), linearly polarized light is transmitted through the compensator (a quarter wave plate) that is an anisotropic optical element with two axis of transmission, one is fast and the other is slow for the transmitted light ${ }^{12}$. The two components of $\mathbf{E}$, which were in phase (for linearly polarized light), emerge out of phase from the plate (for elliptically polarized light). Although ellipsometers that include compensators are useful e.g. for analyzing transparent substrates, many kinds of spectroscopic ellipsometers do not require a compensator.

Figure 3 illustrates how a beam of linearly polarized light incident (i) on a bare surface and a film-covered surface is reflected (r). The plane of incidence (POI) includes the incident beam and the sample surface normal $\mathrm{N}$, which is in the plane of the paper. The components parallel $\left(\mathrm{E}_{p}\right)$ and perpendicular $\left(\mathrm{E}_{s}\right)$ to the POI are out of phase after reflection, and both phase and amplitude of the reflected light changes in a distinct way depending on the optical properties of the surface ${ }^{2}$.

a)

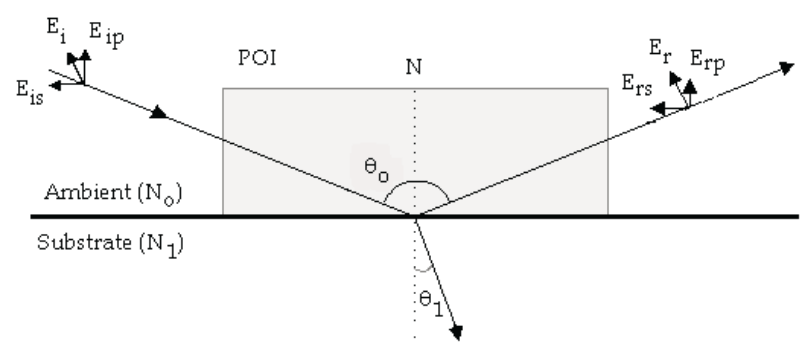

b)

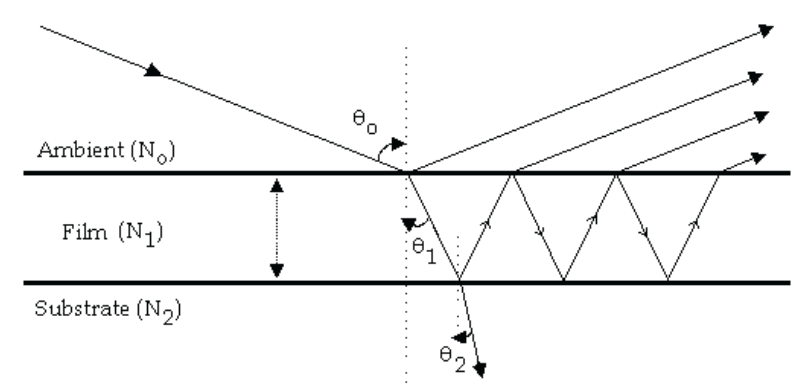

Figure 3. Reflection of polarized light: a) bare surface b) film-covered surface

An ellipsometric measurement allows one to quantify the phase difference between $\mathrm{E}_{p}$ and $\mathrm{E}_{s}, \Delta$, and the change in the ratio of their amplitudes given by $\tan \Psi$. For a bare reflecting surface, the forms for $\Delta$ and $\Psi$ are:

$\Delta=\delta_{\mathrm{rp}}-\delta_{\mathrm{rs}}$ and $\tan \Psi=\frac{\left|\mathrm{r}_{p}\right|}{\left|\mathrm{r}_{s}\right|}$,

where $\mathrm{r}_{p}$ and $\mathrm{r}_{s}$ are the Fresnel coefficients for the components $p$ and $s$ of light. The expressions for $\mathrm{r}_{p}$ and $\mathrm{r}_{s}$ consider a single interface between medium 0 (ambient), with a complex refractive index $\mathrm{N}_{0}$, and medium 1 (substrate), with a complex refractive index $N_{1}$ as follows:

$r_{01}=\frac{E_{r p}}{E_{i p}}=\left|r_{p}\right| e^{\left(i \delta_{r p}\right)}=\frac{N_{1} \cos \theta_{0}-N_{0} \cos \theta_{1}}{N_{1} \cos \theta_{0}+N_{0} \cos \theta_{1}}$

A similar expression can be written for $\mathrm{r}_{\mathrm{s}}$, where $\delta_{\mathrm{rp}}$ and $\delta_{\mathrm{rs}}$ are the phase changes for the $p$ and $s$ components. The total reflection coefficient (reflectance) for a film-covered (single) surface at the angle of incidence, $\theta_{0}$, is $\mathrm{R}$ : 
$\mathrm{R}_{\mathrm{p}}=\left|\mathrm{r}_{\mathrm{p}}\right|^{2} \quad \mathrm{R}_{\mathrm{s}}==\left|\mathrm{r}_{\mathrm{s}}\right|^{2}$,

which for multiple interfaces (such as ambient/film/substrate) is:

$R_{p}=\frac{r_{01 p}+r_{12 p} e^{(-i 2 \beta)}}{1+r_{01 p} r_{12 p} e^{(-i 2 \beta)}}$

A similar expression can be written for $R_{s}$, where $\beta$ expresses the film thickness (L) (here medium 1) and the complex refractive index at the wavelength of incident light, $\lambda$ :

$\beta=2 \pi\left(\frac{L_{1}}{\lambda}\right) \mathrm{N}_{1} \cos \theta_{0}$

The resulting change in polarization after reflection from a surface can be measured through a complex reflection coefficient, $r$ :

$\rho=\tan (\Psi) \mathrm{e}^{(\mathrm{i} \Delta)}=\frac{\mathrm{r}_{\mathrm{p}}}{\mathrm{r}_{\mathrm{s}}}$,

which for a model substrate/film/ambient is obtained as

$\rho=\mathrm{f}\left(\mathrm{N}_{0}, \mathrm{~N}_{1}, \mathrm{~N}_{2}, \lambda, \mathrm{L}_{1}, \theta_{0}\right)$,

where $\mathrm{N}_{0}, \mathrm{~N}_{1}$ and $\mathrm{N}_{2}$ are the indexes of refraction for the ambient, film and substrate, respectively. Usually, $\mathrm{N}_{0}, \lambda$ and $\theta_{0}$ are known, $\mathrm{N}_{2}$ can be independently obtained, and $\mathrm{N}_{1}$ and $\mathrm{L}_{1}$ can be obtained.

The complex refractive index $\mathrm{N}$ represents the real and imaginary parts of the refractive index:

$\mathrm{N}=\mathrm{n}+\mathrm{ik}$

where $\mathrm{n}=$ refractive index and $\mathrm{k}=$ damping constant, which describe a change in the phase velocity and amplitude, respectively, of the electromagnetic wave propagating through a medium. For transparent materials, due to the high penetration depths of light, the $\mathrm{k}$ values are very close to zero and $\mathrm{N}=\mathrm{n}$. However, for many other materials, $\mathrm{n}$ and $\mathrm{k}$ are not constant parameters, but they are dependent of $\lambda$, an effect known as dispersion. The penetration depth, $\mathrm{W}$, is defined as the distance at which the magnitude of the electric field decreases $1 / \mathrm{e}$ of its original value ${ }^{13}$ :

$\mathrm{W}=\frac{\mathrm{c}}{2 \omega \mathrm{k}}=\frac{\lambda}{4 \pi \mathrm{k}}$

The inverse of $\mathrm{W}$ is called absorption coefficient, $\alpha$.

Other important physical parameter obtained by ellipsometry is the complex dielectric function, $\varepsilon$, which correlates the optical and electrical properties of materials and provides a sensitive estimate of surface conditions. As obtained by ellipsometry, $\varepsilon$ is expressed in terms of the complex reflection coefficient, $\rho$, for a simple ambient/ film (f)/substrate (s) optical model:

$\frac{\varepsilon_{\mathrm{s}}}{\varepsilon_{\mathrm{f}}}=\sin ^{2} \theta_{0}+\sin ^{2} \theta_{0} \tan ^{2} \theta_{0}\left(\frac{1-\rho}{1+\rho}\right)$,

This equation is applied for homogeneous, isotropic substrates. For an overlayer on a substrate, $\varepsilon$ is represented by $\langle\varepsilon>$, the pseudodielectric function constant with real and imaginary parts, where the brackets indicate a mixture:

$<\varepsilon>;\left\langle\varepsilon_{1}>+\mathrm{i}<\varepsilon_{2}>;<\varepsilon>=\tilde{\mathrm{N}}^{2} ; \varepsilon_{1}=\mathrm{n}^{2}-\mathrm{k}^{2}\right.$; and $\varepsilon_{2}=2 \mathrm{nk}$
Of particular importance is the $<\varepsilon_{2}>$ spectrum since it indicates the absortivity of a medium through the damping constant, k. Further discussion will appear below in the section on examples.

The dielectric function of materials is related to the composition and microstructure of an essentially inhomogeneous phase ${ }^{14}$. When subjected to an external applied electric field, E, this phase can be represented as a mixture of polarizable species and empty space ${ }^{14-16}$. For this, at least two constituents contribute to this representation with their atomic polarizabilities, $\alpha_{1}$ and $\alpha_{2}$, as such related to the dipole moment (p) of each constituent as p $=\alpha \mathbf{E}_{\text {loc }} . \mathbf{E}_{\text {loc }}$ is the local $\mathbf{E}$ at an atomic entity represented as a polarized sphere embedded in a continuous, insulating medium with a dielectric constant, $\varepsilon$, where $\mathbf{P}$ is the polarization density (dipole moment per volume unit in a spherical cavity, Lorentz model):

$\mathbf{E}_{\mathrm{loc}}=\mathbf{E}+\frac{4 \pi}{3} \mathbf{P} ;$ where $\mathbf{P}=\frac{\varepsilon-1}{4 \pi} \mathbf{E} ;$

The microscopic quantity $\alpha$ can be correlated to the macroscopic quantity $\varepsilon$ by the well-established Clausius-Mosotti equation ${ }^{15}\left(\mathrm{~N}_{\mathrm{i}}=\right.$ density of the $i^{\text {th }}$ polarizable species):

$\frac{\varepsilon-1}{\varepsilon+2}=\frac{4 \pi}{3} \mathrm{~N}_{\mathrm{i}} \alpha$

The same equation can be rewritten for a heterogeneous medium with components 1 and 2 yielding the Lorentz-Lorenz (LL) effective medium expression:

$\frac{\varepsilon-1}{\varepsilon+2 \varepsilon}=\mathrm{f}_{1} \frac{\varepsilon_{1}-1}{\varepsilon_{1}+2}+\mathrm{f}_{2} \frac{\varepsilon_{2}-1}{\varepsilon_{2}+2}$,

where $f_{i}$ is the relative volume fraction of the $i^{\text {th }}$ species. The LL approximation is appropriate for a gas phase host (free space) surrounding spheres as inclusions. The general expression derived by Maxwell Garnett (M-G) in terms of a predominant host matrix (dense phase) with a dielectric constant $\varepsilon_{\mathrm{h}}$ and inclusions of ' 1 ' fully surrounded by ' 2 ' is:

$\frac{\varepsilon-\varepsilon_{\mathrm{h}}}{\varepsilon+2 \varepsilon_{\mathrm{h}}}=\mathrm{f}_{1} \frac{\varepsilon_{1}-\varepsilon_{\mathrm{h}}}{\varepsilon_{1}+2 \varepsilon_{\mathrm{h}}}+\mathrm{f}_{2} \frac{\varepsilon_{2}-\varepsilon_{\mathrm{h}}}{\varepsilon_{2}+2 \varepsilon_{\mathrm{h}}}$,

The model most used in ellipsometry for analyzing surfaces and films is the Bruggeman effective medium approximation (BEMA), which is similar to the M-G model but considers the host medium as an aggregate or a random mixture of dense phases of inclusions $(\varepsilon=$ $\left.\varepsilon_{\mathrm{h}}, \mathrm{f}_{1} \approx \mathrm{f}_{2}\right)$ :

$0=\mathrm{f}_{1} \frac{\varepsilon_{1}-\varepsilon}{\varepsilon_{1}+2 \varepsilon}+\mathrm{f}_{2} \frac{\varepsilon_{2}-\varepsilon}{\varepsilon_{2}+2 \varepsilon}$

The effective medium approximations are very important for optical modeling, particularly for describing microscopic roughness. The comparison between generated (modeled) and experimental results is made through a fitting procedure. A good model is constructed from the chemical and physical histories of a system in which differences between the chosen model and experimental data are minimal. The mean-squared error (MSE) is the quality estimator of parameters as a sum of squares of differences between modeled and measured data ${ }^{17}$ :

MSE $=\frac{1}{2 N-M} \sum_{i=1}^{N}\left(\frac{\Psi_{i}^{\bmod }-\Psi_{i}^{\exp }}{\sigma_{\Psi, i}^{\exp }}\right)^{2}+\left(\frac{\Delta_{i}^{\bmod }-\Delta_{i}^{\exp }}{\sigma_{\Delta, i}^{\exp }}\right)^{2}$, 
where $\mathrm{N}$ is the number of $(\Psi, \Delta)$ pairs, $\mathrm{M}$ is the number of variable parameters in the chosen model, and $\mathrm{s}$ is the standard deviation of the $i^{\text {th }}$ experimental data point. A regression algorithm (for example the Lavenberg-Marquardt algorithm) is then applied in order to calculate and adjust the variable parameters from model and experimental data to a minimum value of MSE.

\section{INSTRUMENTATION}

A typical ellipsometer requires a source of collimated unpolarized light, a polarizer, an analyzer, possibly a compensator, and a photodetector. The compensator if used can be positioned before or after reflection from a surface for a null ellipsometer. In this case, the polarizer and analyzer optical elements are rotated with the sample in phase until extinction of light is achieved. Figure 4 shows a typical configuration for a single (wavelength) ellipsometer with a sequence of polarizer, compensator, sample, and analyzer known as PCSA ellipsometer ${ }^{8}$.

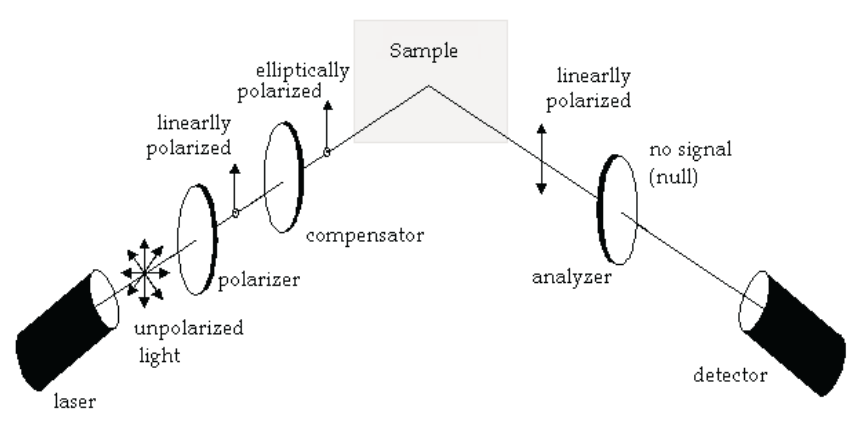

Figure 4. Schematic diagram of a polarizer-compensator-sample-analyzer (PCSA) ellipsometer

The unpolarized light source is converted to linearly polarized light after passing through the polarizer. The compensator (usually a quarter-wave plate fixed at $45^{\circ}$ ) imparts a change of phase, and elliptically polarized light is produced before incidence on the sample. It can be shown that using a quarter-wave plate at $45^{\circ}$ to the POI, all polarization states can be obtained merely by rotating the polarizer. The sample imparts, in general, elliptically polarized light, and then, the PCS combination can be made to yield linear polarized light merely by rotating $\mathrm{P}$. The linearly polarized light produced by the
PCS combination can be totally extinguished with the analyzer and detected (usually by a photomultiplier, PMT). The complex reflection coefficient, $r$, is obtained by varying manually the azimuths of $\mathrm{P}$ and $A$ for a fixed $C$ until there is no detection of light. At this point, the values of $\mathrm{P}, \mathrm{A}$, and $\mathrm{C}$ are related to $\Delta$ and $\Psi$ from which $\mathrm{r}$ is obtained.

Rotating-element instruments became widely used for spectroscopic measurements after the development of digital computers $^{8,18}$. The spectroscopic ellipsometer determines $\Psi$ and $\Delta$ as a function of $\lambda$, and requires a monochromator, or spectrometer, and a light detector. One example of a rotating analyzer ellipsometer (RAE) is shown in Figure 5. The incident energy range for these instruments is usually between 1.5 and $6.0 \mathrm{eV}$, however spectroscopic ellipsometers in the UV-vis-infrared region are presently available.

The RAE requires a light source, a fixed polarizer, a rotating analyzer, and a detector. After reflection from a surface, the light upon emerging from the analyzer has a time varying intensity that is detected. The mechanical parts of RAE have two optical arms, which can be rotated within the plane of incidence of light typically between 45 and $90^{\circ}$. The light beam (a Xe high pressure lamp) is collimated by a lens before reaching the polarizer. An electric shutter is used in order to be able to subtract external light. Both polarizer and analyzer are a GlanTaylor type - two calcite prisms coupled on their hypotenuse faces. The analyzer is rotated at a frequency w using a motor. A telescope is used for optical alignment. The first element of detection is an optical fiber, which carries the signal without any interference from room light to a monochromador coupled with a PMT tube ${ }^{19}$.

The RAE uses the Fourier analysis to obtain $\alpha$ and $\beta$ (Fourier coefficients), which describe the phase and relative amplitude of the ac component of the varying light flux transmitted from the analyzer. The intensity waveform obtained from the ellipsometric measurement has a sinusoidal form given by:

$I(t)=I_{0}(1+\alpha \cos 2 A+\beta \sin 2 A)$,

where: $\mathrm{A}=2 \pi \omega \mathrm{t} ; \alpha=\frac{\tan ^{2} \Psi-\tan ^{2} \mathrm{P}}{\tan ^{2} \Psi+\tan ^{2} \mathrm{P}} ; \beta=\frac{2 \tan P \cos \Delta \tan \Psi}{\tan ^{2} \Psi+\tan ^{2} \mathrm{P}}$

$\mathrm{I}_{0}$ is the intensity that is obtained by averaging the measured intensity (I) over a number of analyzer revolutions, $A(t)$ is the instantaneous azimuth of the analyzer transmission axis, and $\omega$ is the angular frequency of the rotating element. The Fourier coefficients are related to the ellipsometric parameters $\Psi$ and $\Delta$ as follows ${ }^{19}$ :

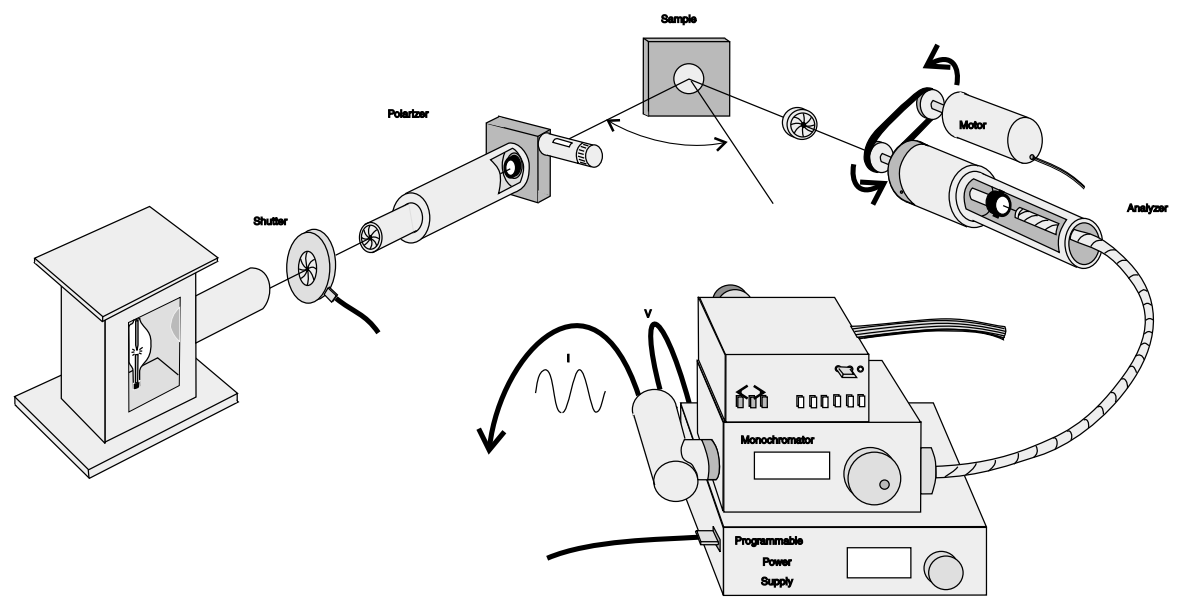

Figure 5. Schematic diagram of a polarizer-sample-rotating analyzer ellipsometer 
$\tan \Psi=\tan P \sqrt{\frac{1+\alpha}{1-\alpha}} \quad \cos \Delta=\frac{\beta}{\sqrt{1-\alpha^{2}}}$,

where $\mathrm{P}$ is the polarization azimuth that is obtained by the maximum and minimum intensity of light upon emerging the rotating analyzer.

\section{APPLICATIONS}

\section{$\mathrm{Si} / \mathrm{SiO}_{2}$ system}

For several decades, Si has been the most widely used semiconductor in microelectronics mainly because $\mathrm{SiO}_{2}$ films can be readily grown on the $\mathrm{Si}$ surface, and the resulting $\mathrm{Si} / \mathrm{SiO}_{2}$ interface has shown a low interface electronic state that enables device operation. This is called electronic passivation, and it has not been realized to the same extent with any other semiconductor/dielectric combination. Although oxidation of $\mathrm{Si}$ occurs naturally in air, high-quality oxides with controllable properties and thickness are usually prepared by the thermal oxidation of extremely clean single crystal $\mathrm{Si}$ wafers at $900-1000{ }^{\circ} \mathrm{C}^{20}$.

The $\mathrm{Si} / \mathrm{SiO}_{2}$ interface has been extensively studied using ellipsometry. As an example, ellipsometric results have indicated that an intermediate layer exists as $\mathrm{SiO}_{\mathrm{x}}{ }^{21,22}$ between an atomically abrupt $\mathrm{Si}$ and $\mathrm{SiO}_{2}$ interface. However, the influence of this intermediate layer is negligible for modeling of thicker films as compared with a few nanometers thick $\mathrm{SiO}_{\mathrm{x}}{ }^{23}$. The database for a $\mathrm{SiO}$ layer is tabulated ${ }^{24}$, and both $\mathrm{Si} / \mathrm{SiO}_{2}$ and $\mathrm{Si} / \mathrm{SiO} / \mathrm{SiO}_{2}$ models can be chosen for a best fitting of ellipsometric data.

Another important example is the possibility of obtaining $<\varepsilon>$ for Si covered with a contaminant overlayer or a microscopic rough layer. It is expected that $\langle\varepsilon\rangle$ decreases for modified surfaces due to a depolarization effect of the incident light beam. Since the penetration depths of light into different materials varies considerably from $\mathrm{nm}$ to $\mathrm{cm}$, an overlayer with only $0.1 \mathrm{~nm}$ can affect substantially $\rho,<\varepsilon>$, and $\mathrm{R}^{25}$. This phenomenon has been explained for transparent and rough overlayers ${ }^{25}$.

The incident light transverses a transparent overlayer and reflects at the layer-substrate interface. After multiple reflections, the light beam that enters the detector is retarded with respect to one of the component of polarized light. As such, if only the $s$-component is totally reflected, the $p$-component is retarded inasmuch as it travels further. This small phase change can be detected by an ellipsometer, and different $\Delta / \Psi$ trajectories and lower values of $<\varepsilon>$ are obtained. Concerning a rough layer, the light beam is depolarized after reflections due to light scattering from imperfections.

The presence of a transparent overlayer or a microscopic rough layer can be misinterpreted for Si because $<\varepsilon>$ decreases in a similar way for both modified surfaces ${ }^{25}$. In this case, complementary techniques are necessary for structural characterization. Aspnes has shown that if $\left\langle\varepsilon>\right.$ of the substrate, $\left\langle\varepsilon_{\mathrm{s}}>\right.$, is much larger than $\langle\varepsilon>$ of the overlayer, $<\varepsilon_{0}>$, and $<\varepsilon>$ of ambient is 1 , then $<\varepsilon>$ is given by ${ }^{25,26}$ :

$<\varepsilon>=\varepsilon_{\mathrm{S}}+\frac{4 \pi \mathrm{iL}}{\lambda} \varepsilon_{\mathrm{s}}^{3 / 2}$

This equation shows that $<\varepsilon>$ depends only on $\mathrm{L}$ (overlayer thickness) and not on $\left\langle\varepsilon_{0}\right\rangle$, and this fact allows one to assert the quality of the surface coverage without identifying the nature of the overlayer $^{25}$

In order to illustrate this phenomena, Figure 6 shows the $<\varepsilon>$ spectra of $\mathrm{Si}$ covered with a $\mathrm{SiO}_{2}$ overlayer that has different values of thickness. The $\mathrm{E}_{1}$ and $\mathrm{E}_{2}$ peaks at 3.4 and $4.3 \mathrm{eV}$ are attributed to interband transitions of $\mathrm{Si}$, and they are very sensitive to small variations in surface conditions.

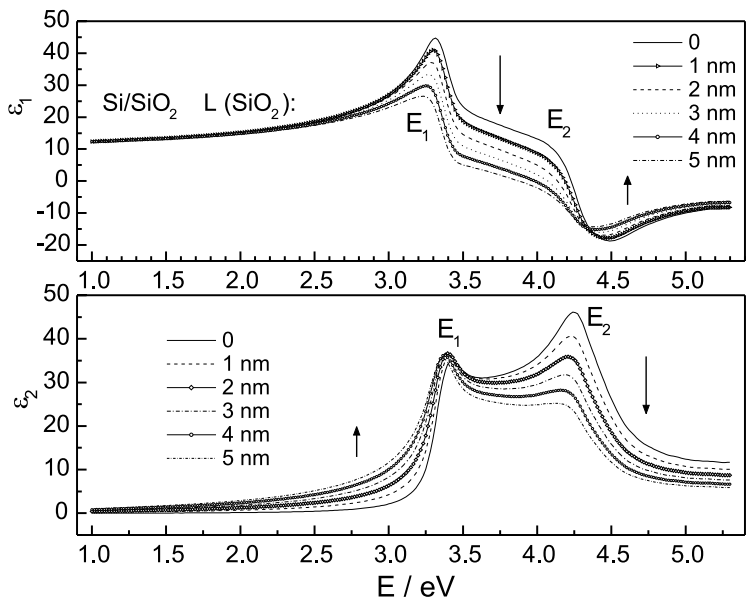

Figure 6. Generated dielectric function of $\mathrm{Si} / \mathrm{SiO}{ }_{2}$ at different values of thickness

As predicted by equation 24 , at a fixed $\mathrm{L}$, the ratio $\mathrm{L} / \lambda$ decreases as $\lambda$ increases, and the main contribution to $\langle\varepsilon\rangle$ is the real part of $<\varepsilon>,<\varepsilon_{1}>\left(\right.$ peak $\left.E_{1}\right)$. On the other hand, as $\lambda$ decreases, $L / \lambda$ increases, and the imaginary part of $\langle\varepsilon\rangle$ becomes more important affecting the values of $<\varepsilon_{2}>\left(\right.$ peak $\left.\mathrm{E}_{2}\right)$.

Although $\mathrm{Si} / \mathrm{SiO}_{2}$ interfaces can be readily modeled, it is impossible to accurately discriminate suboxide $\left(\mathrm{SiO}_{\mathrm{x}}\right)$ layers and microscopic roughness of $\mathrm{Si}$ for ultrathin $\mathrm{SiO}_{2}$ films with thickness values less than $10 \mathrm{~nm}$ as required in advanced integrated circuits ${ }^{27}$. The use of spectroscopic immersion ellipsometry, however, improves the sensitivity of ellipsometry to interfaces by using a transparent liquid with a refractive index that matches to the overlayer eliminating its optical contribution ${ }^{28}$. This technique enhances the interface sensitivity for values of $\Delta$ due to the presence of a liquid by an order of magnitude, and effects such as microscopic roughness can be studied at large areas (as compared with small areas analyzed by atomic force microscopy (AFM), for example).

\section{Optical parameters of bare substrates}

Glass

Although glass is commonly used as a substrate for thin films, its transparency in the visible energy range, and internal reflections, are of concern for ellipsometry. Since the light penetration depth in glasses is high (e.g. $29 \mathrm{~cm}$ at $589.3 \mathrm{~nm}$ for flint glass) ${ }^{13}$, reflection from the backside of a glass slide can be only suppressed either by abrading and/or blackening the backside surface, or by using suitable analysis software, which includes contributions of a second light beam (two reflected beams reach the detector from the top and back glass surfaces $)^{17}$. While the latter solution includes more parameters making it more difficult to obtain a fit, it is an option for thin glass substrates, which are difficult to be abraded without breaking.

The values of $\Delta$ are very high (close to $180^{\circ}$ ) or small (close to $\left.0^{\circ}\right)$ at incident angles $\left(\theta_{0}\right)$ that are below and above, respectively, to the Brewster angle, $\theta_{\mathrm{B}}$, (usually $\theta_{\mathrm{B}}$ is near to $56-57^{\circ}$ for most glasses) ${ }^{29}$. Because of the difficulty in measuring $\Delta$ near $180^{\circ}$ and $0^{\circ}$, the data analysis can be made at different values of $\theta_{0}$ using only the $\Psi$ values and ignoring the $\Delta$ values ${ }^{17}$. However, the best solution for glass substrates is to first experimentally obtain $\theta_{\mathrm{B}}$, and then use a region for analysis where the values of $\Delta$ are different from $0^{\circ}$ and $180^{\circ}$, that is usually between \pm 1.0 from the value of $\theta_{B}$. 
$A u$

Among several examples of substrates, $\mathrm{Au}$ is one of the most often used in ellipsometry, a choice that is justifiable mainly due to its high reflectivity and conductivity, and stability to oxidation as compared with other metals.

Differences in the $<\varepsilon>$ spectra of Au films have been attributed to effects such as surface roughness, overlayers, and voids in the bulk $^{30}$. The influence of a rough surface layer on the $\langle\varepsilon>$ spectra of Au can be observed in Figure 7a, b. A single optical model was assumed (Au/layer/ambient).
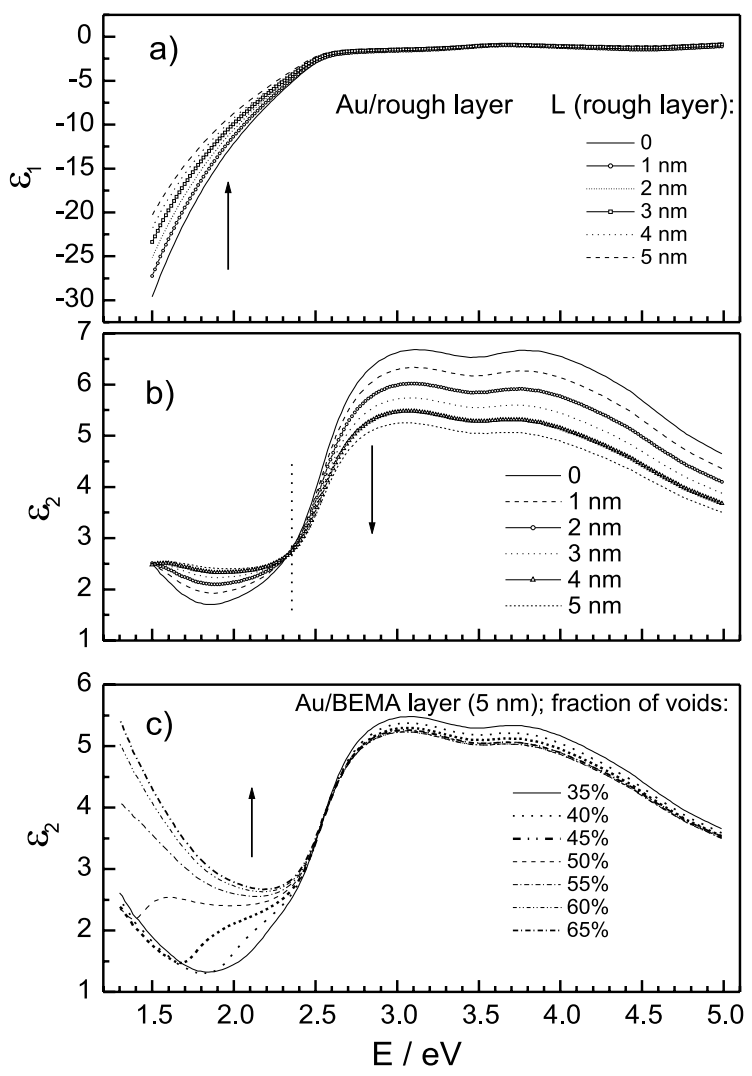

Figure 7. a) and b) Generated dielectric function of Au/surface rough layer (EMA overlayer with $50 \%$ of voids) at different values of thickness; c) Generated imaginary part of the dielectric function of Au/BEMA overlayer at different fractions of voids

The dielectric function of $\mathrm{Au}$ can be split into two regions at a threshold energy of about $2.3 \mathrm{eV}$ (dash line). These regions correspond to intraband transitions below $2.3 \mathrm{eV}$ (optical excitation of an electron below to above the Fermi energy within the same band), and interband transitions above $2.3 \mathrm{eV}$ (direct transition of an electron to another band), which can be described by Drude freeelectron (intraband) and Lorentz oscillator models (interband) ) $^{31,32}$.

Experimentally, it was verified that the $<\varepsilon_{2}>$ spectrum changes drastically in the intraband region for $\mathrm{Au}$ particles dispersed in a polymer ${ }^{33}$ or in a glass ${ }^{34}$ matrix. The same behavior was observed for bulk $\mathrm{Au}$ films with thickness ranging from 10 to $25 \mathrm{~nm}$ and 50 to $500 \mathrm{~nm}$, even after film annealing ${ }^{30,35}$. As shown in Figure 7c, higher concentration of voids induces considerable variation in the $<\varepsilon_{2}>$ spectrum in the intraband region. A BEMA model was assumed for void concentrations ranging from 35 to $65 \%$.

As these results have indicated, ellipsometry is a powerful technique for characterizing Au surfaces, particularly because contaminant overlayers are a common reality for this metal under air exposure. Another topic of current interest is the study of adsorption processes of sulfur-containing monolayers and polymers on pre-treated $\mathrm{Au}$ substrates. Here we only mention that these systems can be readily studied using ellipsometry along with techniques such as cyclic voltammetry, AFM and X-ray photoelectron spectroscopy (XPS)

\section{Polymers}

Ellipsometry has been applied to the study of numerous polymer films, in particular those that are electrochemically obtained on optically smooth electrodes ${ }^{36-38}$. The examples presented here are: study of the conversion reaction of a polysulfonium precursor into poly-p-phenylenevinylene (PPV) films; and optical properties of transparent and absorbing conducting polymer films.

The precursor route is the most common method used to prepare light-emitting PPV films ${ }^{39}$. For this methodology, cast, spin-coat, or Langmuir-Blodgett films of a soluble precursor, poly- $p$-xylylidene tetrahydrothiophenium chloride, PTHT, can be prepared on different substrates ${ }^{40}$. These films are converted into PPV films under vacuum at around $200-230^{\circ} \mathrm{C}$ by eliminating sulfide groups. The effects of the thermal treatment on the optical properties of PTHT films deposited on Au can be observed in Figure $8^{41}$.

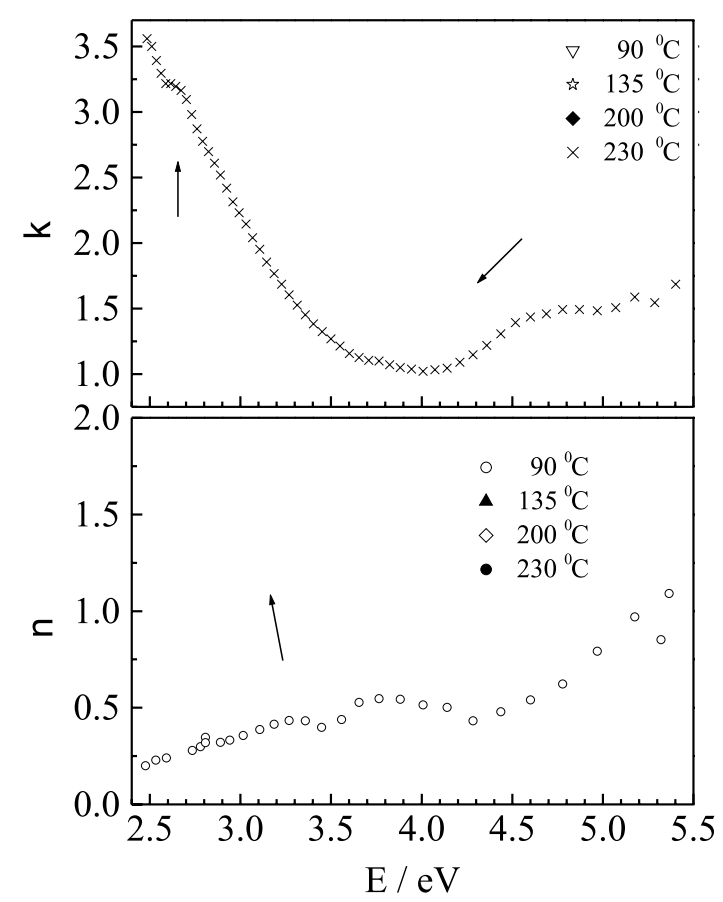

Figure 8. Optical parameters ( $k$ and $n$ ) measured for PTHT films converted into PPV films at different temperatures

The variation of $\mathrm{n}$ and $\mathrm{k}$ for an ambient/film/substrate single optical model indicates that the chain conjugation of PTHT increases at temperatures higher than $90^{\circ} \mathrm{C}$. In particular, since $\mathrm{k}$ is directly related to the absorption coefficient, films converted at higher temperatures present higher values of $k$. The conversion process of PTHT into PPV can be followed using ellipsometry and more details can be obtained using structural characterization techniques. Considering the optical properties of a transparent polymer, the modeling is straightforward in a wide range of energy. The film used for exemplifying this analysis is a polymethacrylate derivative with a pendent anthracene chomophore, poly(methylmethacrylate-co-9-anthracenyl-methylmethacrylate (4:1), 
MDCPAC $^{42}$. The absorption spectrum of this polymer shows the typical bands of anthracene between 300 to $400 \mathrm{~nm}$; at longer wavelengths, the film is transparent. Figure 9 shows $\Psi$ and $\Delta$ spectra of a MDCPAC film deposited by spin coating on Au-coated substrates ${ }^{43}$.

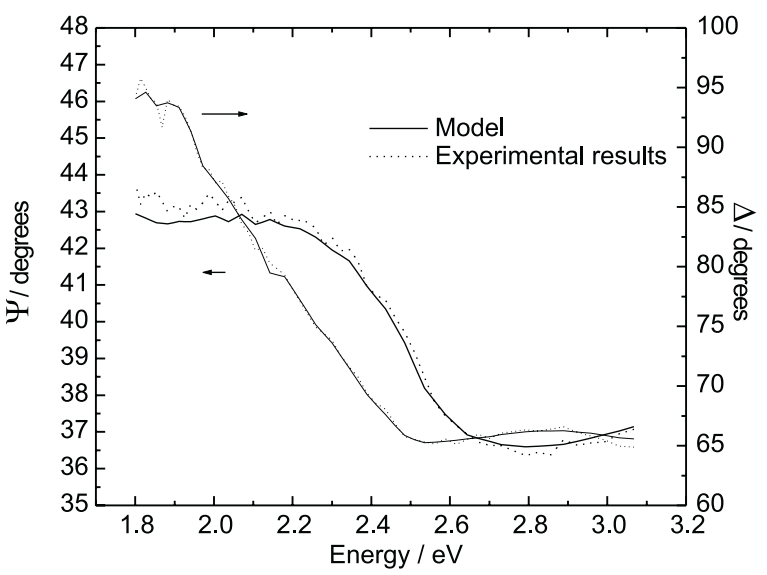

Figure 9. Experimental and modeled (Au/Cauchy layer) values of $Y$ and $D$ for a MDCPAC film on Au-coated substrate

A single optical model was chosen as Au/Cauchy layer/ambient, the film thickness (L), and A, B, and C parameters of the Cauchy equation were fitted (the Cauchy equation is an empirical formula that parameterizes the optical properties of materials). The thickness obtained for this film was $23.5 \pm 0.4 \mathrm{~nm}$ through an excellent fitting in the spectral region of 400 to $700 \mathrm{~nm}$ (about 3.1 to $1.8 \mathrm{eV}$ ). The values of $\mathrm{n}$ and $\mathrm{k}$ of the MDCPAC film can be also obtained using this model.

The use of ellipsometry for characterizing absorbing films demands more effort from the ellipsometer user. One method used for obtaining $\mathrm{L}, \mathrm{n}$ and $\mathrm{k}$ of absorbing polymer films is the multiplesample analysis. A thicker film with a known value of $L$ (as obtained by profilometry or AFM, for example) is analyzed by ellipsometry. Since $\mathrm{L}$ is known, the optical properties of the film can be fitted, and a new database is created. However, this method assumes that the optical properties are identical for all samples, which is not always the case. Other type of analysis is to find a range of transparency of films. A unique solution for optical properties and $\mathrm{L}$ is found only for films that are semi-transparent at about 1.5 and $6.0 \mathrm{eV}$. A relatively new method called Arwin-Aspnes (A-A) method ${ }^{44}$ has been proposed for analyzing several materials including polymers. In this method, different values of $\mathrm{L}$ are assumed, and the proper $\mathrm{L}$ is that one when the substrate-related features are not visibly seen in the $<\varepsilon>$ spectra. Limitations are if the film and substrate have peaks in the same spectral region, or the substrate spectrum is featureless ${ }^{45}$. A solution for finding both optical properties and $\mathrm{L}$ of absorbing films depends much from sample to sample, and talent and dedication (and luck!) of the user.

\section{FINAL REMARKS}

We presented in this paper basic concepts and examples of applications of ellipsometry. The description of models and results is in a simplified form, but the reader can find more details about theoretical and experimental parts in the cited references. The applications of ellipsometry in Materials Science are uncountable, however, the domain of this technique demands time (experience) of the user. Erroneous conclusions can be made without proper data analysis. Besides, highquality samples and complementary techniques of characterization are certainly helpful tools to obtain satisfactory results.

\section{ACKNOWLEDGMENT}

The authors acknowledge support by the National Science Foundation (NSF). D. Gonçalves acknowledges the Brazilian agency FAPESP for a fellowship.

\section{REFERENCES}

1. Irene, E.A.; Thin Solid Films 1993, 233, 96.

2. Irene, E.A.; Woolam, J.A.; Mater. Res. Soc. Bulletin 1995, 20, 24.

3. Drude, P.; The Theory of Optics, Dover: New York, 1959 (from the last English translation in 1902).

4. Rothen, A.; Rev. Sci. Instrum. 1945, 16, 26.

5. Hauge, P.S.; Dill, F.H.; Ellipsometric Thickness Analyzer, IBM J. Res. Develop. 1973, 472.

6. de Nijs, J.M.M.; Holtslag, A.H.M.; Hoeksta, A.; van Silfhout, A; J. Opt. Soc. Am. A 1988, 5, 1466 .

7. Azzam, R.M.A.; Bashara, N.M.; J. Opt. Soc. Am. 1971, 61, 1236.

8. Azzam, R.M.A.; Bashara, N.M.; Ellipsometry and Polarized Light, $2^{\text {nd }}$ ed., Elsevier: Amsterdam, 1987.

9. Azzam, R.M.A.; Selected Papers on Ellipsometry, SPIE Optical Eng. Press, 1991.

10. Hecht, E.; Optics, $2^{\text {nd }}$ ed., Addison-Wesley, 1989.

11. Fowles, G.R.; Introduction to Modern Optics, $2^{\text {nd }}$ ed., Dover: New York, 1975.

12. Pedrotti, F.L.; Pedrotti, L.S.; Introduction to Optics, $2^{\text {nd }}$ ed., Prentice Hall: New Jersey, 1993.

13. Hummel, R.E.; Electronic Properties of Materials, $2^{\text {nd }}$ ed., Springer-Verlag: Berlin, 1993.

14. Aspnes, D.E.; Thin Solid Films 1982, 89, 249.

15. Böttcher, C.J.F.; Theory of Electric Polarization, Elsevier: Amsterdam, 1952.

16. Landauer, R.; Electrical Conductivity in Inhomogeneous Media, Am. Inst. of Phys., 1978, p. 2-45.

17. Woollam, J.A. Co., Inc.; Guide to using WVASE32 software for optical data analysis.

18. Tompkins, H.G.; A User's Guide to Ellipsometry, Academic Press: Boston, 1993.

19. Kim, S.Y.; Spanos, L.; Irene, E.A.; J. Korean Phys. Soc. 1995, 28, 420.

20. Irene, E.A.; Crit. Rev. Solid State Mater. Sci. 1988, 14, 175.

21. Aspnes, D.E.; Theeten, J.B.; Phys. Rev. Lett. 1979, 43, 1046.

22. Aspnes, D.E.; Theeten, J.B.; J. Electrochem. Soc. 1980, 127, 1359.

23. Jellison Jr., G.E.; J. Appl. Phys. 1991, 69, 7627.

24. Philipp, H.R. In Handbook of Optical Constants of Solids; Palik, E.D., ed.; Academic Press: Orlando, 1985, vol. 1, p. 768.

25. Aspnes, D.E. In Optical Properties of Solids: New Developments; Seraphin, B.O., ed.; North-Holland: Amsterdam, 1976, p. 799.

26. Aspnes, D.E. In Handbook of Optical Constants of Solids; Palik, E.D., ed.; Academic Press: Orlando, vol. 1, 1985, p. 89.

27. Wang, Y.; Irene, E.A.; J. Vac. Sci. Technol., B 2000, 18, 279.

28. Zhao, C.; Lefebvre, P.R.; Irene, E.A.; Thin Solid Films 1998, 313-314, 286.

29. The $\theta_{\mathrm{B}}$ is that angle where the reflected light is totally $s$-polarized (only a small fraction of the $p$-component is reflected, $\mathrm{r} \approx 0$ ).

30. Aspnes, D.E.; Kinsbron, E.; Bacon, D.D.; Phys. Rev. B 1980, 21, 3290.

31. Wooten, F.; Optical Properties of Solids, Academic Press: New York, 1972.

32. Abèles, F., ed.; Optical Properties of Solids, North-Holland: Amsterdam, 1972.

33. Dalacu, D.; Martinu, L.; J. Appl. Phys. 2000, 87, 228.

34. Kreibig, U.; Solid State Commun. 1978, $28,767$.

35. Thèye, M.-L.; Phys. Rev. B 1970, 2, 3060.

36. Barbero, C.; Kötz, R.; J. Electrochem. Soc. 1994, 141, 859.

37. Kim, Y.-T.; Collins, R.W.; Vedam, K.; Allara, D.L.; J. Electrochem. Soc. 1991, 138, 3266.

38. Cruz, C.M.G.S.; Ticianelli, E.A.; J. Electroanal. Chem. 1997, 428, 185

39. Massardier, V.; Guyot, A.; Tran, V.H.; Polymer 1994, 35, 1561.

40. Marletta, A.; Gonçalves, D.; Oliveira Jr., O.N.; Faria, R.M.; Guimarães, F.E.G.; Adv. Mater. 2000, 12, 69.

41. Bianchi, R.F.; Gonçalves, D.; Faria, R.M.; Irene, E. A.; unpublished work.

42. Ma, D.; Aguiar, M.; Freire, J.A.; Hümmelgen, I.A.; Adv. Mater. 2000, 12, 1063.

43. Gonçalves, D.; Aguiar, M.; Akcelrud, L.; Irene, E. A.; unpublished work.

44. Arwin, H.; Aspnes, D.E.; Thin Solid Films 1984, 113, 101.

45. Järrendall, K.; Arwin, H.; Thin Solid Films 1998, 313-314, 114 